Research Article

\title{
Developing an Extreme Learning Machine-Based Model for Estimating the Isothermal Compressibility of Biodiesel
}

\author{
Yue Wang $\mathbb{D}^{1}$ and Hamid Heydari $\mathbb{D}^{2}$ \\ ${ }^{1}$ School of Intelligent Manufacturing, Xinxiang Vocational and Technical College, Xinxiang, Henan 453003, China \\ ${ }^{2}$ Institute of Petroleum Engineering, University of Tehran, Tehran, Iran \\ Correspondence should be addressed to Yue Wang; wangyue20210601@126.com and Hamid Heydari; h.heydari.2014@ut.ac.ir
}

Received 3 June 2021; Revised 26 June 2021; Accepted 5 July 2021; Published 10 July 2021

Academic Editor: Alireza Baghban

Copyright (c) 2021 Yue Wang and Hamid Heydari. This is an open access article distributed under the Creative Commons Attribution License, which permits unrestricted use, distribution, and reproduction in any medium, provided the original work is properly cited.

\begin{abstract}
Nowadays, the high consumption of fossil fuels has caused many pollutants and environmental problems. Biodiesel has recently been considered as a clean and renewable alternative to fossil fuels. They are found in some molecular structures including fatty acid ethyl esters (FAEEs) and also fatty methyl esters (FAMEs), having various thermophysical characteristics. Thus, it appears essential to select the suitable methods for a particular diesel engine to estimate the ester characteristics. The current research sets out to develop a new and robust method predicting isothermal compressibility of long-chain fatty acid methyl and ethyl esters directly from several basic efficient parameters (pressure, temperature, normal melting point, and molecular weight). Therefore, as a novel and prevailing mathematical method in this field, an extreme learning machine was implemented for isothermal compressibility on the massive dataset. According to statistical evaluations, this novel established model had high accuracy and applicability $\left(R^{2}=1\right.$ and RMSE $\left.=0.0018714\right)$ which is more accurate than previous models presented by former researchers. Among various factors of the sensitivity analysis, temperature and pressure had the greatest effect on the output values, so that the output parameter has a direct relationship with temperature and an inverse relationship with pressure with relevancy factors of $22.44 \%$ and $-79.81 \%$.
\end{abstract}

\section{Introduction}

Conventional hydrocarbon fuels have limited resources and lead to environmental pollution, asphaltene deposition, and formation damage. The rise in the use and contamination of fossil fuels in 2008 by up to 2500 million tons draws the attention of researchers to the quest for better substitutes for common fuels. Biodiesel, among different fuel sources, is more desirable since it may be extricated from available agrarian yields, effortlessly mix (with all amounts) with gasoline, have more agreeable transport and storage, and release a lesser amount of greenhouse gas than usual fossil fuels [1-5].

Often, biodiesel is composed of long-chain FAMEs and FAEEs, generated via transesterification of industrial and natural fatty acid resources, such as vegetable oil and animal fat, besides other fatty feedstock. The long-chain fatty acids' esterification system by short-chain alcohols, including ethanol or methanol, is indicated by the following reaction in the base catalyst existence:

$$
R-\mathrm{COOH}+R^{\prime}-\mathrm{OH} \stackrel{\text { base }}{\leftrightarrow} R-\mathrm{COO}-\mathrm{R}^{\prime}+\mathrm{H}_{2} \mathrm{O},
$$

where $R$ and $\mathrm{R}^{\prime}$ are the long-alkyl and short-alkyl chains, respectively. In an industrial sector, this reaction outlet, in a reactor unit, is a mixture of different kinds of long-chain FAMEs and FAEEs, typically related to impurities. The mixture is well-known as biodiesel. Typical biomass sources of fatty acids in generating biodiesel are rapeseed, soybean, palm oils, and sunflower [6-8]. Various types of long-chain fatty acids can be found in fatty feedstocks from which biodiesel can be obtained, resulting in different molecular structures of fatty acid esters in biodiesel. Different thermophysical characteristics result from various molecular 
structures, which impact the fuel performance, the exhaust gas content, and combustion efficacy in diesel engines [9-11].

Fatty acid esters have four basic thermophysical properties that control the combustion behavior of biodiesel. These characteristics are density, speed of sound, isentropic compressibility, and isothermal compressibility [12, 13]. Appropriate fuel combustion necessitates accurate timing for fuel injection, being thoroughly associated with speed of sound within the biodiesel and compressibility. The higher the speed of sound and compressibility of the biodiesel, the earlier the injection timing, which leads to the release of more nitrogen gas oxide [14-16]. The characteristics studied (density, sound speed, isentropic compressibility, and isothermal compressibility) are the roles of fatty acid esters' pressure, temperature, and molecular structure, which are the major biodiesel components.

To define the biodiesel's performance, information on the stated thermophysical characteristics' values for longchain FAEEs and FAMEs is necessary for a variety of pressure and temperature settings because of their significance and efficient parts. In laboratories, the real values of the measures are provided by experimental measurement. In most cases, these measurements, however, are problematic or could be tedious and costly. Therefore, in this scope, it is essential to use empirical models and mathematical relations.

To establish computational correlations, various statistical-mathematical algorithms (machine-learning methods) exist [17, 18]. The well-known instruments to develop correlation include the adaptive neurofuzzy inference system (ANFIS), genetic programming (GP), artificial neural networks (ANNs), and stochastic gradient boosting (SGB) trees [18-23].

Many studies have been performed for predicting biodiesel characteristics thus far. Several approaches, including the ANFIS and ANN, have been used to investigate and model biodiesel density at a variety of pressures and temperatures [24-29]. Some researchers also have studied sound speed and compressibility $[16,30]$. Nevertheless, the current research aims at proposing a new and appropriate model to predict isothermal compressibility of several long-chain fatty acid esters based on modest and basic parameters, such as pressure, temperature, normal melting point, and molecular weight. In this research, an extreme learning machine (ELM) was applied as a prevailing mathematical modeling method, the use of which appears to be new in this field. The applicability and prediction performance of this novel model has been increased due to using great deals datasets with various temperature and pressure settings. Furthermore, some other benefits of the models are their simplicity, and basic parameters, the high precision.

\section{Materials and Methods}

2.1. Data Collection. In order to build the new model, a large dataset containing 310 data samples of isothermal compressibility was collected from the literature. The relevant collected data are given elsewhere [31]. In practice, this dataset was first divided into two subsets, randomly, as a tool for the development of powerful model based on the data, among which $70 \%$ was regarded as training data, and the remaining parts were considered testing data. The training data were then applied to design the novel model, and the testing data were used to assess the established model's estimation ability. Similar trends for more precision were seen in both data subsets.

2.2. Extended Learning Machine. The extended learning machine or ELM is a hidden-layer single counter neural network that in the updated version may not make the hidden layer of neurons homogeneous [32]. ELM, despite its special features, avoids many problems such as the amount of learning and training courses and the selection of stop criteria [33-35].

The ELM algorithm is developed from biological learning systems and BP learning algorithms that have been proposed to solve difficult problems. Its main application is clustering, regression, classification, and extraction of features. Depending on the characteristics of biological learning, a neural network can have random neurons whose settings are independent of the situation [36, 37]. In the extended learning machine method, all hidden neurons are independent of training samples and each other, which is in contrast to crash training methods/network models [38, 39].

Learning nodes do not need to be regulated, and hidden weights can be determined before the learning phase. Also, when the hidden neurons are large enough for problems, the ELM architecture is strong, but it does not work well for highly data-dependent learning methods.

The extended learning machine method does not require adjustment for the connection between input and hidden layers. The ELM output is a simple model with an output node such as follows [40]:

$$
f_{L}(x)=\sum_{i=1}^{L} \beta_{i} h_{i}(x)=h(x) \beta .
$$

In the above formula, $h(x)=\left[h_{1}(x) \ldots h_{L}(x)\right]$ represents the output vector of the hidden layer, which is dependent on the input of $x$. This function plots the input $d$-dimensional data into the output $L$-dimensional data, and $\beta=\left[\beta_{1} \ldots \beta_{L}\right]^{T}$ is the output weight vector between the output node and the hidden layer. The ELM decision function is described as a classification of binary applications as follows [41]:

$$
f_{L}(x)=\operatorname{sign}[h(x) \beta] .
$$

The training error in ELM is the lowest compared to the old methods and even tries to reach the smallest norm of output weight.

From Bartlett's theory, we can see that for a similar training error compared to training neural networks, lower weights are required to achieve better generalization performance. It should be noted that if $h(x)$ is an infinitely variable activation function, then there is no need to adjust the ELM weight [42]. 
At the beginning of the training phase, the input weight can be selected randomly. Finally, in the training phase, the weight of the input layer is fixed. Also, the weight that connects the hidden and output layers is the weight that searches the least squares of the following target function. The ELM method reduces output weight and learning error norms as follows [40]:

$$
\text { Minimize : }\left\|H \beta-T^{2}\right\| \text { and }\|\beta\|,
$$

where $H$ represents the output layer activation matrix of the hidden layer.

Simultaneous minimization of the weight connection norm $b$ and learning error is the main task of the extended learning machine method. From solving the least squares problem, the output weights of the hidden layer can be easily calculated as follows $[43,44]$ :

$$
\beta=H^{\dagger} T \text {. }
$$

In the above equation, $H^{\dagger}$ stands for the pseudoinverted Moore-Penrose $H$ matrix. The ELM method consists of several steps, which are summarized in the following:

(1) Randomly set hidden node parameters for hidden nodes, input weight, biases $b_{\mathrm{i}}$, and $a_{\mathrm{i}}$

(2) Obtain the output layer of the hidden layer

(3) Calculate the output weight vector

$$
\beta=H^{\dagger} T \text {. }
$$

$T$ is defined as follows:

$$
T=\left[T_{1} \ldots T_{L}\right]^{T} .
$$

Analyzing the quasi-inverse Moore-Penrose of a matrix can be performed in several ways, including the orthogonalization and iterative methods, the ultrasonic projection method, and unit value analysis. The orthogonal projection method is possible in the following two ways: for times when $H H^{T}$ is nonsingular and $H^{\dagger}=H^{T}\left(H H^{T}\right)^{-1}$ or for times $H^{T} H$ and $H^{\dagger}=H^{T}\left(H^{T} H\right)^{-1}$ is nonsingular. This solution works more accurately and its generalized performance is better as well [45].

From ELM theory, it can be concluded that many $h(x)$ feature mapping functions may be used in ELM design. As a result, any continuous operation can be approximated by ELM.

\section{Results and Discussion}

In Table 1, we used different methods including STD, $R^{2}$, MSE, RMSE, and MRE\% to analyze the estimated isothermal compressibility and compare it with the real value. To calculate the statistical parameters, we have used the following equations.

$$
\begin{aligned}
\text { MRE } & =\frac{1}{n} \sum_{i=1}^{n} \frac{\left|y_{\text {exp. } i}-y_{\text {pred. } i}\right|}{y_{\text {pred. } i}}, \\
\text { MSE } & =\frac{1}{n} \sum_{i=1}^{n}\left(y_{\text {exp. } i}-y_{\text {pred. } i}\right)^{2}, \\
\text { RMSE } & =\sqrt{\operatorname{MSE}}=\sqrt{\frac{1}{n} \sum_{i=1}^{n}\left(y_{\text {exp. } i}-y_{\text {pred. } . i}\right)^{2}}, \\
\text { STD } & =\sqrt{\frac{1}{n-1} \sum_{i=1}^{n}\left(\frac{y_{\text {exp. } i}-y_{\text {pred. } i}}{y_{\text {exp. } . i}}\right)^{2}}, \\
R^{2} & =1-\frac{\sum_{i=1}^{n}\left(y_{\text {pred. } i}-y_{\text {exp. } .}\right)^{2}}{\sum_{i=1}^{n}\left(y_{\text {pred. } . i}-\overline{y_{\text {exp } . i}}\right)^{2}} .
\end{aligned}
$$

In equations (8)-(12), $y_{\text {exp. } i}$ and $y_{\text {pred.i } i}$ represent the desired value and the estimated values, respectively, and the parameter $n$ represents the number of experimental data. Models with more $R^{2}$ and less STD, MSE, and MRE\% are more accurate in evaluating output values. Looking at Table 1, it is easy to see that the GPR model is an accurate model in estimating isothermal compressibility. Figure 1 also shows that according to the relative deviation analysis, this model has a high accuracy in predicting the target values, and as shown in the figure, most relative deviation points are close to zero and its maximum value is equal to $2.5 \%$.

One method of evaluating the accuracy of the model in predicting target values is to visually compare real data and estimated data. Figure 2 deals with such comparison. Accordingly, the prediction of the majority of data related to the testing and training phases is done by the ELM model with high precision.

Figure 3 shows the cross-plot of estimated isothermal compressibility related to the ELM model and experimental ones. Since the slope of the line is near to $45^{\circ}$, the correctness of the presented model is high, and $R^{2}$ values related to this model for training and testing phases were obtained as 0.9999 and 0.9998 , respectively.

By examining the statistical parameters, it can be seen that the performance of the current model is better in predicting target values compared to the previously suggested models. By comparing the results of this study with the study of Abooali et al. that used the same data to estimate the isothermal compressibility, the superiority of the ELM model can be concluded [31]. Table 2 proves the accuracy and efficiency of the models. The results of estimation of the isothermal compressibility of diesel oil by ELM, SGB, and GP methods are given. As shown, the developed model has better accuracy compared to the previous models in the 
TABLE 1: Calculated values of statistical data in order to evaluate the target parameter using the ELM model.

\begin{tabular}{lcccccc}
\hline Parameter & Dataset & $R^{2}$ & MRE $(\%)$ & MSE & RMSE & STD \\
\hline & Train & 1.000 & 0.13 & 0.0000019 & 0.0013666 \\
Isothermal compressibility $(1 / \mathrm{GPa})$ & Test & 1.000 & 0.18 & 0.0000084 & 0.0029067 & 0.0011408 \\
& Total & 1.000 & 0.14 & 0.0000035 & 0.0018714 & 0.0016566 \\
\hline
\end{tabular}

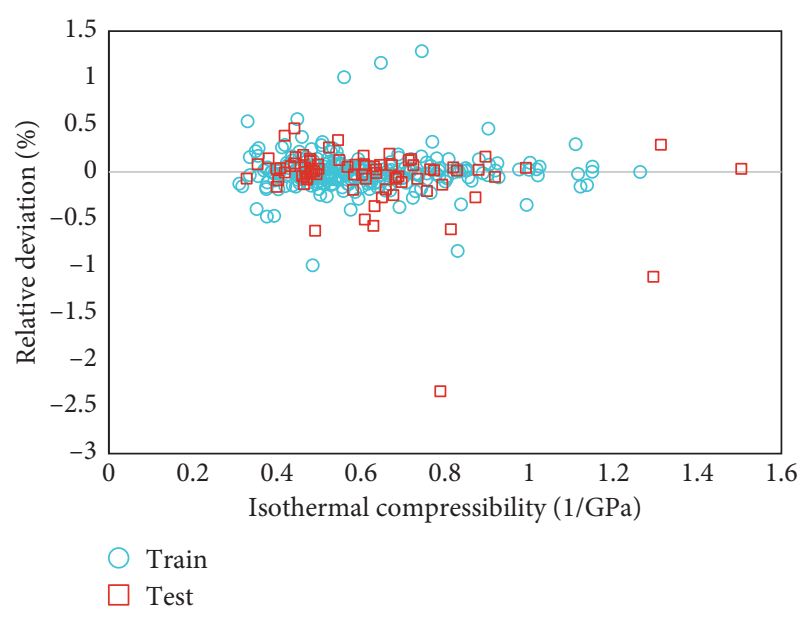

FIGURE 1: Relative deviation analysis performed on training and testing data.

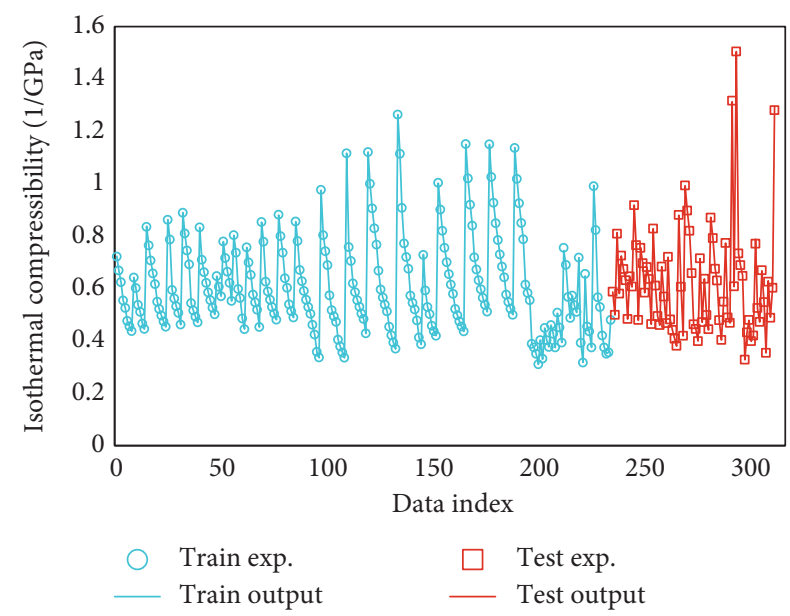

FIGURE 2: Estimated isothermal compressibility values in comparison with experimental data using the ELM model.

testing phase. The value of $R^{2}$ is 0.9998 for the developed model and 0.99931 and 0.99885 for the previous models.

3.1. Sensitivity Analysis. To investigate the input parameters that affect the isothermal compressibility, the following equation can be used to calculate the relevancy factor.

$$
r=\frac{\sum_{i=1}^{n}\left(X_{k i}-\overline{X_{k}}\right)\left(Y_{i}-\bar{Y}\right)}{\sqrt{\sum_{i=1}^{n}\left(X_{k i}-\overline{X_{k}}\right)^{2} \sum_{i=1}^{n}\left(Y_{i}-\bar{Y}\right)^{2}}} .
$$

In equation (13), $\bar{Y}$ represents the mean output, $Y_{i}$ abbreviated $i^{\text {th }}$ output and $X_{k i}$ is the $k^{\text {th }}$ input, and the input is represented by $X_{k}$. The value of $r$ is consistently less than

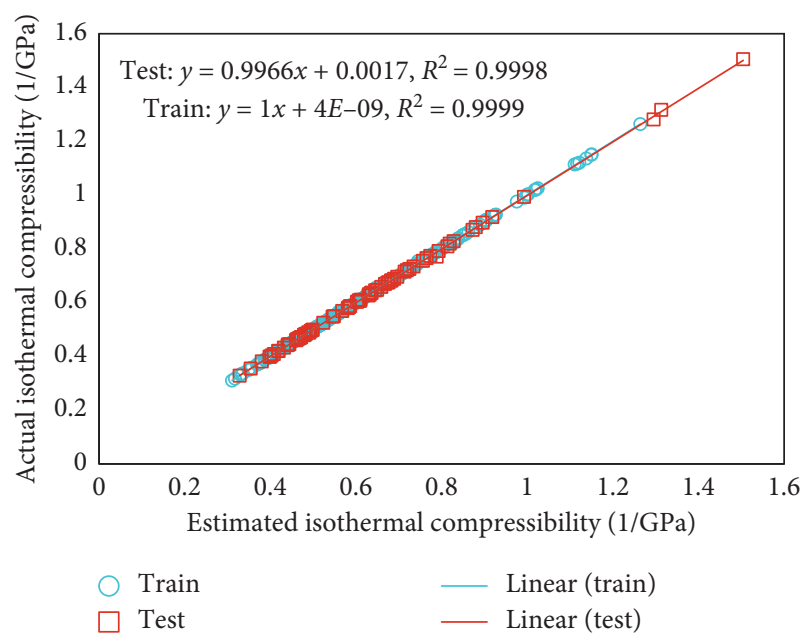

FIGURE 3: Linear regression for the prediction of isothermal compressibility using the ELM model in the training and testing datasets.

TABle 2: Comparison of the results of Abooali et al.'s models with the current model in predicting output values at the testing phase.

\begin{tabular}{lccc}
\hline & \multicolumn{1}{c}{ Abooali et al.'s models } & Current work \\
\hline Model & SGB & GP & ELM \\
$R^{2}$ & 0.99931 & 0.99885 & 0.9998 \\
\hline
\end{tabular}

unity. Figure 4 shows the impact of each of the input parameters on the isothermal compressibility. It can be seen from the presented figure that the pressure and melting point have a negative effect on the output. Unlike the negative effect of them, the two characteristics of temperature and molecular weight have a positive effect on the target values. In fact, the isothermal compressibility increases with increasing temperature $(T)$ and molecular weight (MW) of biodiesel oils. Also, it can be seen that temperature has the most effect and pressure has the least effect on the isothermal compressibility.

3.2. Detecting Available Outlier Datapoints in the Dataset. Data measured in the laboratory are always subject to an error. To check the accuracy of real data, we used the leverage limit method. Due to its critical impact in evaluating the proposed model, based on soft computing, the approach presented in this method is underlying and useful. The main purpose of this method is to ensure that the real output datapoints are authentic. In this research, Williams's design has been used to evaluate the feasibility and reliability of the developed ELM model to predict target values [46]. According to this concept, the data that are found in the 


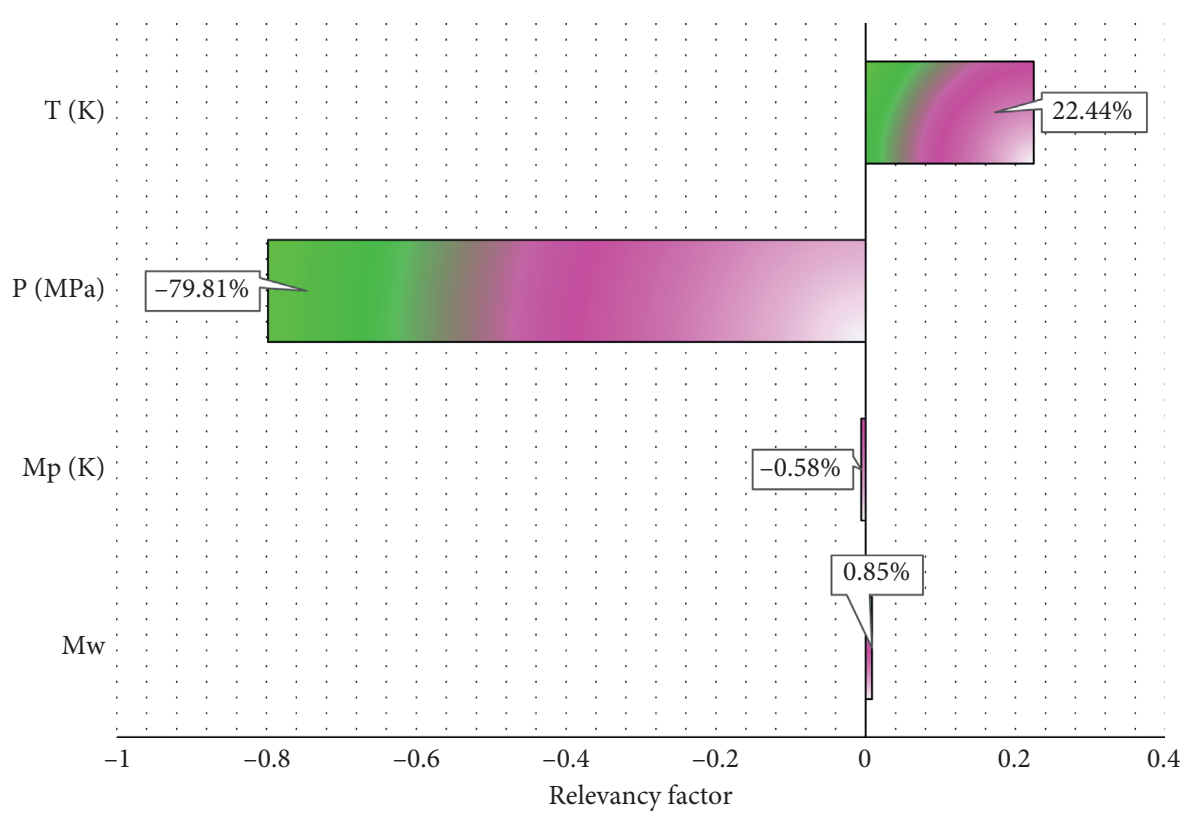

FIgURE 4: Sensitivity analysis for determining the impact of input parameters on the isothermal compressibility.

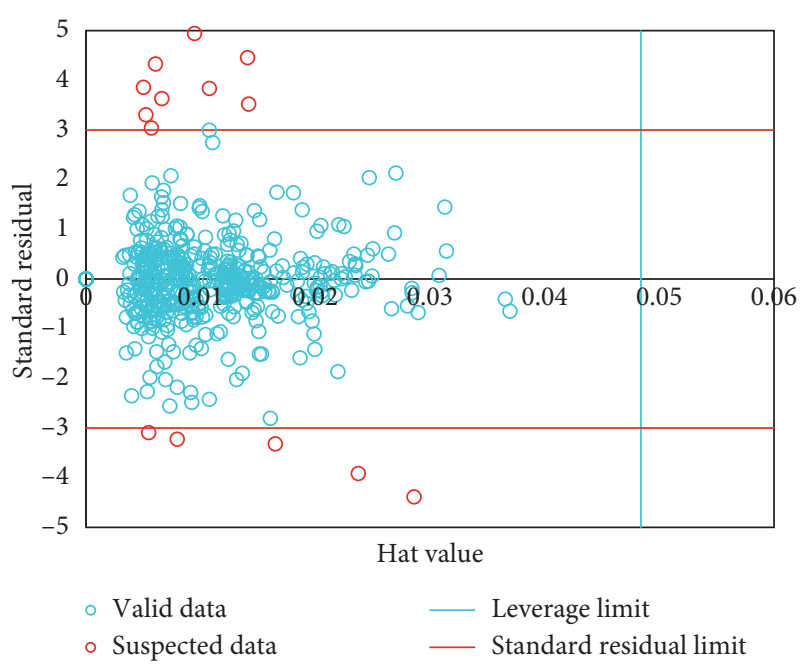

FIGURE 5: Checking suspected datapoints using the leverage limit method.

range of $0 \leq H \leq H^{*}$ and $-3 \leq$ standardized residual $\leq 3$ are reliable. As a result, for the proposed model, it can be stated that the satisfactory points of the upper lever are in the range of $H \leq 0.04$ for biodiesel oil data. In contrast, some points that are not in the range of 3 and -3 can be taken into account as the functional domain of the model used. Based on the results of the ELM model presented in this study, there are only 14 data in the datapoints outrange of biodiesel oil. This phase is illustrated in Figure 5.

\section{Conclusion}

This study aimed in establishing a new model for estimating the isothermal compressibility as a collection of central characteristics of long-chain fatty acid esters. To establish this model, a dataset including 310 data was implemented.
The modeling procedure was designed on the basis of analogous independent parameters, including temperature $(\mathrm{T})$, pressure $(\mathrm{P})$, normal melting point $(\mathrm{Tm})$, and molecular weight $(\mathrm{Mw})$ of fatty acid esters. The current research used the prevailing statistical-mathematical machine-learning frameworks of an extreme learning machine. The results show that the established model was appropriate and valid in estimating the hinted characteristics concerning statistical variables. Nevertheless, more evaluations indicated the precision of the ELM technique to be higher than that of previously proposed model. This model offers novel uses in simulating controlling biodiesel systems, besides estimating the characteristics for newly developed long-chain fatty acid esters.

\section{Data Availability}

The data used to support the findings of this study are included within the article.

\section{Conflicts of Interest}

The authors declare that they have no conflicts of interest.

\section{References}

[1] B. Kegl and S. Pehan, "Influence of biodiesel on injection, fuel spray, and engine characteristics," Thermal Science, vol. 12, no. 2, pp. 171-182, 2008.

[2] H. Fukuda, A. Kondo, and H. Noda, "Biodiesel fuel production by transesterification of oils," Journal of Bioscience and Bioengineering, vol. 92, no. 5, pp. 405-416, 2001.

[3] R. M. Balabin, E. I. Lomakina, and R. Z. Safieva, "Neural network (ANN) approach to biodiesel analysis: analysis of biodiesel density, kinematic viscosity, methanol and water contents using near infrared (NIR) spectroscopy," Fuel, vol. 90, no. 5, pp. 2007-2015, 2011. 
[4] M. Sun, L. Yan, L. Zhang, L. Song, J. Guo, and H. Zhang, "New insights into the rapid formation of initial membrane fouling after in-situ cleaning in a membrane bioreactor," Process Biochemistry, vol. 78, pp. 108-113, 2019.

[5] H. Zhang, M. Sun, L. Song, J. Guo, and L. Zhang, "Fate of $\mathrm{NaClO}$ and membrane foulants during in-situ cleaning of membrane bioreactors: combined effect on thermodynamic properties of sludge," Biochemical Engineering Journal, vol. 147, pp. 146-152, 2019.

[6] E. H. I. Ndiaye, M. Habrioux, J. A. P. Coutinho, M. L. L. Paredes, and J. L. Daridon, "Speed of sound, density, and derivative properties of methyl oleate and methyl linoleate under high pressure," Journal of Chemical \& Engineering Data, vol. 58, no. 8, pp. 2345-2354, 2013.

[7] M. A. Aissa, G. R. Ivaniš, I. R. Radović, and M. L. Kijevčanin, "Experimental investigation and modeling of thermophysical properties of pure methyl and ethyl esters at high pressures," Energy \& Fuels, vol. 31, no. 7, pp. 7110-7122, 2017.

[8] S. Baroutian, M. K. Aroua, A. A. A. Raman, and N. M. N. Sulaiman, "Density of palm oil-based methyl ester," Journal of Chemical \& Engineering Data, vol. 53, no. 3, pp. 877-880, 2008.

[9] M. Dzida, S. Jężak, J. Sumara, M. Żarska, and P. Góralski, "High-pressure physicochemical properties of ethyl caprylate and ethyl caprate," Journal of Chemical \& Engineering Data, vol. 58, no. 7, pp. 1955-1962, 2013.

[10] A. S. Silitonga, H. H. Masjuki, T. M. I. Mahlia, H. C. Ong, W. T. Chong, and M. H. Boosroh, "Overview properties of biodiesel diesel blends from edible and non-edible feedstock," Renewable and Sustainable Energy Reviews, vol. 22, pp. 346360, 2013.

[11] Y.-C. Su, Y. A. Liu, C. A. Diaz Tovar, and R. Gani, "Selection of prediction methods for thermophysical properties for process modeling and product design of biodiesel manufacturing," Industrial \& Engineering Chemistry Research, vol. 50, no. 11, pp. 6809-6836, 2011.

[12] K. Anand, R. Sharma, and P. S. Mehta, "A comprehensive approach for estimating thermo-physical properties of biodiesel fuels," Applied Thermal Engineering, vol. 31, no. 2-3, pp. 235-242, 2011.

[13] P. Saxena, S. Jawale, and M. H. Joshipura, "A review on prediction of properties of biodiesel and blends of biodiesel," Procedia Engineering, vol. 51, pp. 395-402, 2013.

[14] S. V. D. Freitas, Â. Santos, M.-L. C. J. Moita et al., "Measurement and prediction of speeds of sound of fatty acid ethyl esters and ethylic biodiesels," Fuel, vol. 108, pp. 840-845, 2013.

[15] S. V. D. Freitas, D. L. Cunha, R. A. Reis et al., "Application of wada's group contribution method to the prediction of the speed of sound of biodiesel," Energy \& Fuels, vol. 27, no. 3, pp. 1365-1370, 2013.

[16] S. V. D. Freitas, M. L. L. Paredes, J.-L. Daridon, Á. S. Lima, and J. A. P. Coutinho, "Measurement and prediction of the speed of sound of biodiesel fuels," Fuel, vol. 103, pp. 1018-1022, 2013.

[17] M. Soleimani Lashkenar, B. Mehdizadeh, and K. Movagharnejad, "Application of genetic algorithm based support vector machine model in second virial coefficient prediction of pure compounds," Iranian Journal of Chemistry and Chemical Engineering (IJCCE), vol. 37, no. 5, pp. 189-198, 2018.

[18] R. Maachou et al., "Energy consumption modeling in activated sludge process using coupling PCA-ANFIS approach," Iranian Journal of Chemistry and Chemical Engineering (IJCCE), vol. 38, no. 6, pp. 261-273, 2019.
[19] D. Abooali and E. Khamehchi, "Estimation of dynamic viscosity of natural gas based on genetic programming methodology," Journal of Natural Gas Science and Engineering, vol. 21, pp. 1025-1031, 2014.

[20] N. Saghatoleslami et al., "A neuro-fuzzy model for a dynamic prediction of milk ultrafiltration flux and resistance," Iranian Journal of Chemistry and Chemical Engineering (IJCCE), vol. 26, no. 2, pp. 53-61, 2007.

[21] S. Zahedi Abghari and A. Imani, "Determination of suitable operating conditions of fluid catalytic cracking process by application of artificial neural network and firefly algorithm," Iranian Journal of Chemistry and Chemical Engineering (IJCCE), vol. 37, no. 6, pp. 157-168, 2018.

[22] H. Mirshahvalad et al., "A neural networks model for accurate prediction of the flash point of chemical compounds," Iranian Journal of Chemistry and Chemical Engineering (IJCCE), vol. 39, no. 4, pp. 297-304, 2020.

[23] K. Akbarzade and I. Danaee, "Nyquist plots prediction using neural networks in corrosion inhibition of steel by schiff base," Iranian Journal of Chemistry and Chemical Engineering (IJCCE), vol. 37, no. 3, pp. 135-143, 2018.

[24] S. O. Giwa, S. O. Adekomaya, K. O. Adama, and M. O. Mukaila, "Prediction of selected biodiesel fuel properties using artificial neural network," Frontiers in Energy, vol. 9, no. 4, pp. 433-445, 2015.

[25] Y. Kassem and H. Çamur, "Prediction of biodiesel density for extended ranges of temperature and pressure using adaptive neuro-fuzzy inference system (ANFIS) and radial basis function (RBF)," Procedia Computer Science, vol. 120, pp. 311-316, 2017.

[26] M. Lapuerta, J. Rodríguez-Fernández, and O. Armas, “Correlation for the estimation of the density of fatty acid esters fuels and its implications. A proposed biodiesel cetane index," Chemistry and Physics of Lipids, vol. 163, no. 7, pp. 720-727, 2010.

[27] S. Phankosol, K. Sudaprasert, S. Lilitchan, K. Aryusuk, and K. Krisnangkura, "Estimation of density of biodiesel," Energy \& Fuels, vol. 28, no. 7, pp. 4633-4641, 2014.

[28] A. A. Refaat, "Correlation between the chemical structure of biodiesel and its physical properties," International Journal of Environmental Science \& Technology, vol. 6, no. 4, pp. 677694, 2009.

[29] C. I. Rocabruno-Valdés, L. F. Ramírez-Verduzco, and J. A. Hernández, "Artificial neural network models to predict density, dynamic viscosity, and cetane number of biodiesel," Fuel, vol. 147, pp. 9-17, 2015.

[30] P. Krisanangkura, S. Lilitchan, K. Aryusuk, and K. Krisnangkura, "A mathematical model for estimating molecular compressibility of fatty-acid methyl ester and biodiesel," Journal of the American Oil Chemists' Society, vol. 96, no. 1, pp. 3-14, 2019.

[31] D. Abooali, R. Soleimani, and S. Gholamreza-Ravi, "Characterization of physico-chemical properties of biodiesel components using smart data mining approaches," Fuel, vol. 266, Article ID 117075, 2020.

[32] G.-B. Huang, "An insight into extreme learning machines: random neurons, random features and kernels," Cognitive Computation, vol. 6, no. 3, pp. 376-390, 2014.

[33] A. Akusok, K.-M. Bjork, Y. Miche, and A. Lendasse, "Highperformance extreme learning machines: a complete toolbox for big data applications," IEEE Access, vol. 3, pp. 1011-1025, 2015.

[34] G.-B. Huang, Q.-Y. Zhu, K. Z. Mao, K. S. Chee, P. Saratchandran, and N. Sundararajan, "Can threshold 
networks be trained directly?" IEEE Transactions on Circuits and Systems II: Express Briefs, vol. 53, no. 3, pp. 187-191, 2006.

[35] G.-B. Huang, L. Chen, and C.-K. Siew, "Universal approximation using incremental constructive feedforward networks with random hidden nodes," IEEE Transactions on Neural Networks, vol. 17, no. 4, pp. 879-892, 2006.

[36] D. L. Sosulski, M. L. Bloom, T. Cutforth, R. Axel, and S. R. Datta, "Distinct representations of olfactory information in different cortical centres," Nature, vol. 472, no. 7342, pp. 213-216, 2011.

[37] G.-B. Huang, Q.-Y. Zhu, and C.-K. Siew, "Extreme learning machine: a new learning scheme of feedforward neural networks," in Proceedings of the 2004 IEEE International Joint Conference on Neural Networks (IEEE Cat. No. 04CH37541), Budapest, Hungary, July 2004.

[38] M.-B. Li, G.-B. Huang, P. Saratchandran, and N. Sundararajan, "Fully complex extreme learning machine," Neurocomputing, vol. 68, pp. 306-314, 2005.

[39] N.-Y. Liang, G.-B. Huang, P. Saratchandran, and N. Sundararajan, "A fast and accurate online sequential learning algorithm for feedforward networks," IEEE Transactions on Neural Networks, vol. 17, no. 6, pp. 1411-1423, 2006.

[40] G.-B. Huang, "Extreme learning machine for regression and multiclass classification," IEEE Transactions on Systems, Man, and Cybernetics, Part B (Cybernetics), vol. 42, no. 2, pp. 513-529, 2011.

[41] T. O. Owolabi, "Extreme learning machine and swarm-based support vector regression methods for predicting crystal lattice parameters of pseudo-cubic/cubic perovskites," Journal of Applied Physics, vol. 127, no. 24, Article ID 245107, 2020.

[42] T. O. Owolabi and M. A. Abd Rahman, "Prediction of band gap energy of doped graphitic carbon nitride using genetic algorithm-based support vector regression and extreme learning machine," Symmetry, vol. 13, no. 3, p. 411, 2021.

[43] M. A. Mujtaba, H. H. Masjuki, M. A. Kalam et al., "Ultrasound-assisted process optimization and tribological characteristics of biodiesel from palm-sesame oil via response surface methodology and extreme learning machine - cuckoo search," Renewable Energy, vol. 158, pp. 202-214, 2020.

[44] S. Rashidi, M. Mehrad, H. Ghorbani et al., "Determination of bubble point pressure \& oil formation volume factor of crude oils applying multiple hidden layers extreme learning machine algorithms," Journal of Petroleum Science and Engineering, vol. 202, Article ID 108425, 2021.

[45] N. Nabipour, A. Mosavi, A. Baghban, S. Shamshirband, and I. Felde, "Extreme learning machine-based model for Solubility estimation of hydrocarbon gases in electrolyte solutions," Processes, vol. 8, no. 1, p. 92, 2020.

[46] M. H. Ahmadi, A. Baghban, M. Sadeghzadeh, M. Hadipoor, and M. Ghazvini, "Evolving connectionist approaches to compute thermal conductivity of $\mathrm{TiO} 2 /$ water nanofluid," Physica A: Statistical Mechanics and Its Applications, vol. 540, Article ID 122489, 2020. 